Research Article

\title{
Cytoprotective Effects of Citicoline and Homotaurine against Glutamate and High Glucose Neurotoxicity in Primary Cultured Retinal Cells
}

\author{
Sergio Davinelli, Flavia Chiosi, Roberto Di Marco, Ciro Costagliola, and Giovanni Scapagnini \\ Department of Medicine and Health Sciences "V. Tiberio", University of Molise, Campobasso, Italy \\ Correspondence should be addressed to Sergio Davinelli; sergio.davinelli@unimol.it
}

Received 7 July 2017; Accepted 27 September 2017; Published 15 October 2017

Academic Editor: Deborah A. Ferrington

Copyright $\odot 2017$ Sergio Davinelli et al. This is an open access article distributed under the Creative Commons Attribution License, which permits unrestricted use, distribution, and reproduction in any medium, provided the original work is properly cited.

\begin{abstract}
Citicoline and homotaurine are renowned compounds that exhibit potent neuroprotective activities through distinct molecular mechanisms. The present study was undertaken to demonstrate whether cotreatment with citicoline and homotaurine affects cell survival in primary retinal cultures under experimental conditions simulating retinal neurodegeneration. Primary cultures were obtained from the retina of fetal rats and exposed to citicoline plus homotaurine $(100 \mu \mathrm{M})$. Subsequently, neurotoxicity was induced using excitotoxic levels of glutamate and high glucose concentrations. The effects on retinal cultures were assessed by cell viability and immunodetection of apoptotic oligonucleosomes. The results showed that a combination of citicoline and homotaurine synergistically decreases proapoptotic effects associated with glutamate- and high glucose-treated retinal cultures. This study provides an insight into the potential application of citicoline and homotaurine as a valuable tool to exert neuroprotective effects against retinal damage.
\end{abstract}

\section{Introduction}

Visual impairment is a worldwide health problem affecting about 285 million people [1]. Recently, it was estimated that with aging populations in high-income regions of Central/ Eastern Europe, diabetic retinopathy and glaucoma will become the most important causes of vision loss [2]. The key cell type implicated in the development of glaucoma and diabetic retinopathy is the retinal ganglion cell (RGC), and apoptosis of RGC is the final event leading to visual loss $[3,4]$. The cause of apoptosis is excitotoxicity due to excessive synaptic glutamate activity. Glutamate is one of the major excitatory neurotransmitters in the brain and exists in high concentrations in the retina. It is thought that exposure to moderately elevated levels of glutamate can trigger cellular processes in neurons that eventually lead to apoptosis [5, 6]. In addition, increasing evidence shows that several neuronal cell types in the retina are highly susceptible to hyperglycemia-mediated apoptosis [7]. Cell culture models have substantially contributed to the characterization of the pathophysiology of retinal neurodegeneration, providing a simplified tool to investigate in an isolated context the detrimental effects of high glucose (HG) concentrations and an excessive amount of glutamate $[8,9]$. In recent years, research efforts have been made to identify neuroprotective drugs able to prevent visual field loss and preserve visual function. However, the failure of recent clinical trials raised several doubts regarding the strategies to achieve neuroprotection in retinal degeneration [10]. Based on the results of the latest investigations, it is reasonable to assert that a single constituent that affects one target has limited efficacy in preventing the progression of multifactorial diseases. A large body of research revealed that the use of a combination of compounds with synergistic multitarget effects may offer a more powerful approach for disease prevention, including retinal neurodegeneration [11-15]. This study investigated whether cotreatment of citicoline and homotaurine exhibits synergistic neuroprotective effects in experimental conditions associated with neuroretinal degeneration such as glutamate-induced excitotoxicity and HG-induced neurotoxicity. Citicoline (cytidine- $5^{\prime}$-diphosphocholine) is an intermediate in the synthesis of phosphatidylcholine, a component of cell 
membranes. It has been shown that citicoline produces neuroprotective effects in a variety of central nervous system (CNS) injury models, particularly cerebral ischemia [16]. At the experimental level, it has been reported that citicoline is a neuroprotective molecule acting through mechanisms relevant to glaucoma and diabetic retinopathy. The effects proposed to explain the neuroprotective actions of citicoline have been thoroughly reviewed and include antiapoptotic effects, neurotrophic properties, protection after partial optic nerve crush, reduction of excitotoxicity, effects on nonglutamatergic neurotransmitter systems, and effects on remyelination [17]. In recent human studies, citicoline appears to be a promising agent to improve cognitive impairment [18]. Homotaurine (3-aminopropanesulfonate), an analogue of 4 -aminobutyrate ( $\gamma$-aminobutyric acid, GABA), is a small natural aminosulfonate compound identified in different species of marine red algae and then chemically synthesized and introduced into clinical use under the name of tramiprosate [19]. It has been shown that homotaurine may interfere with several cellular pathways, both in vitro and in vivo experimental models, and exert neuroprotective and neurotropic activities through different mechanisms including effects against the oxidative damage to DNA, antifibrillogenic activity, and antinociceptive and analgesic activities. More interestingly, beyond its neuroprotective and neurotropic effects related to the activation of GABA type A receptors, it has been observed that homotaurine prevents the neurotoxicity of $\mathrm{A} \beta$ peptide by reducing amyloid aggregation $[20,21]$. Considering the distinct chemical properties of citicoline and homotaurine, the purpose of this study was to assess whether cotreatment of these compounds may exert synergistic neuroprotective effects on primary retinal cultures.

\section{Materials and Methods}

2.1. Retinal Cultures. The study has been approved by the appropriate ethics committee and has therefore been performed in accordance with the ethical standards laid down in the 1964 Declaration of Helsinki and its later amendments. Primary cultures were obtained from the retinas of fetal Wistar rats (18-19 days' gestation), following a procedure described elsewhere [22]. Briefly, retinal tissues were mechanically dissociated, and the cell suspensions were plated into $60 \mathrm{~mm}$ dish $\left(0.8-1.0 \times 10^{6}\right.$ cells $\left./ \mathrm{mL}\right)$ (Corning, Acton, MA). Retinal cultures were incubated in Eagle's minimal essential medium (MEM) containing $2 \mathrm{mM}$ glutamine, penicillin-streptomycin $(100 \mathrm{U} / \mathrm{ml}, 50 \mu \mathrm{g} / \mathrm{mL})$, and $25 \mathrm{mM} \mathrm{N}$-(2-hydroxyethyl) piperadine- $\mathrm{N}^{\prime}$-(2-ethanesulfonic acid) (HEPES) under an atmosphere of $5 \% \mathrm{CO}_{2}$ in the air. The medium was supplemented with $10 \%$ heat-inactivated fetal bovine serum during the 1st week and with $10 \%$ horse serum for the remaining 8-11 days. To eliminate nonneuronal cells, $10 \mu \mathrm{M}$ cytosine arabinoside (Sigma, St. Louis, MO) was added to the culture. Only those cultures maintained for 9-11 days in vitro and only isolated cells were used in this study. Previous studies using cultured rat retinal cells demonstrated that cell viability was reduced by exposure to glutamate $(1 \mathrm{mM})$ for $10 \mathrm{~min}$. Followed by postincubation in a glutamate-free medium for more than 1 hour $[23,24]$. It was also showed that there was no significant difference between the values of reduction in cell viability between 1-hour and 24-hour incubations [25]. In the present study, glutamate neurotoxicity was assessed using a $25 \mathrm{~min}$ exposure to $100 \mu \mathrm{M}$ glutamate followed by a 24 -hour incubation in the glutamate-free medium. In the second series of experiments, the cells were treated with HG concentrations to mimic the diabetic condition and produce a hyperglycemic insult. When cells reached $80 \%$ confluence, the culture medium was supplemented with glucose, reaching a final concentration of $30 \mathrm{mM}$. Retinal cells were exposed to $\mathrm{HG}$ for 96 hours. The concentration of glucose in control conditions was $5 \mathrm{mM}$. Media were changed every 24 hours in all groups.

2.2. Cell Viability. The assay used to assess cell viability in retinal cells was the (3,4,5-dimethylthiazol-2-yl)-2,5diphenyltetrazolium bromide (MTT) reduction assay modified from that of Mosmann [26]. To evaluate the effect of citicoline and homotaurine on cell survival, the cells were subdivided into three groups and treated for 24 hours with $1 \mu \mathrm{M}, 10 \mu \mathrm{M}$, and $100 \mu \mathrm{M}$ of citicoline (Kyowa Hakko Bio Co. Ltd., Tokyo, Japan) and with $1 \mu \mathrm{M}, 10 \mu \mathrm{M}$, and $100 \mu \mathrm{M}$ of homotaurine (Truffini e Reggè Farmaceutici, Milan, Italy). To evaluate the neuroprotective effects of citicoline and homotaurine, cells were treated with citicoline $100 \mu \mathrm{M}$, homotaurine $100 \mu \mathrm{M}$, or citicoline + homotaurine $100 \mu \mathrm{M}, 24$ hours before glutamate treatment and $30 \mathrm{~min}$ before HG treatment. MTT was added to wells at a final concentration of $0.5 \mathrm{mg} / \mathrm{mL}$ for 1 hour at $37^{\circ} \mathrm{C}$. After this time, the medium was removed and reduced MTT (blue formazan product) was solubilized by adding $100 \mu \mathrm{L}$ dimethyl sulfoxide to each well. After agitation of plates for $15 \mathrm{~min}$, the optical density of the solubilized formazan product in each well was measured using an automatic microplate reader (Molecular Devices, Crawley, UK) with a $570 \mathrm{~nm}$ test wavelength and a $690 \mathrm{~nm}$ reference wavelength.

2.3. Apoptotic Cell Death Detection. Apoptosis was determined by using a Cell Death Detection ELISA ${ }^{\text {PLUS }}$ kit (Roche Applied Science, Indianapolis, IN). This photometric enzyme immunoassay provides the quantitative determination of oligonucleosomes generated from the apoptotic cells. After the treatments, cells were washed, harvested, lysed, and centrifuged to remove nuclei, and supernatants were collected. An aliquot of the supernatant from each sample was incubated with immunoreagents in 96-well streptavidin-coated plates on a shaker. After three washes with incubation buffer, the substrate solution was added to each well, and absorbance was read at $405 \mathrm{~nm}$ in a microplate reader. The enrichment of oligonucleosomes released into the cytoplasm was calculated as absorbance of sample cells/absorbance of control cells.

2.4. Statistical Analysis. Data are expressed as the mean \pm standard error of the mean (SEM) of three independent experiments. Statistical significance was determined using one-way analysis of variance (ANOVA), followed 


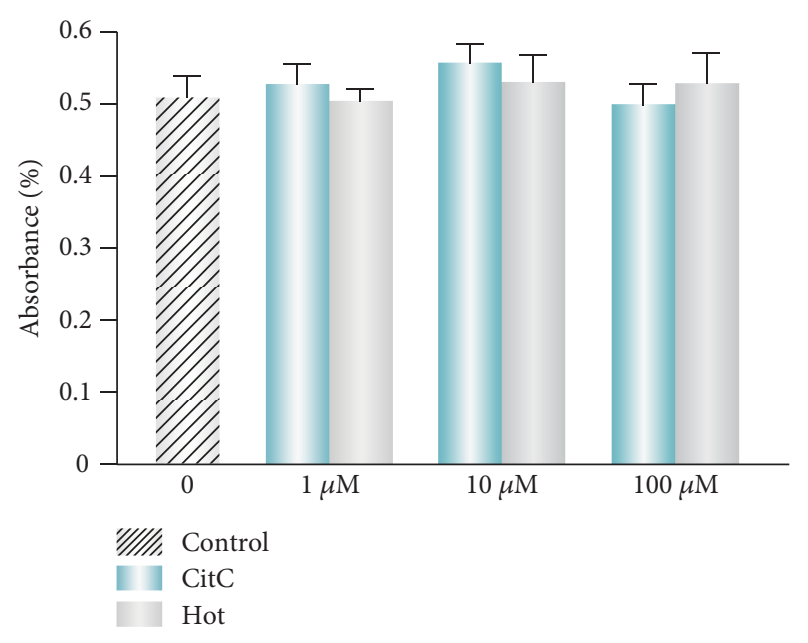

Figure 1: Effects of citicoline and homotaurine on cell viability. MTT assay shows that retinal cells were well preserved in citicoline- or homotaurine-treated cultures, with no evidence of toxicity after treatment at 1,10 , or $100 \mu \mathrm{M}$. All data are represented as the mean \pm SEM of three independent experiments. CitC: citicoline; Hot: homotaurine.

by Tukey's post hoc test. The $p$ value $<0.05$ was considered statistically significant.

\section{Results}

3.1. Viability of Primary Retinal Cultures Is Not Affected by Treatment with Citicoline or Homotaurine. To determine the potential neuroprotective activity of citicoline and homotaurine, we firstly treated retinal cells with increasing concentrations of citicoline or homotaurine for 24 hours. We investigated whether $1 \mu \mathrm{M}, 10 \mu \mathrm{M}$, and $100 \mu \mathrm{M}$ of citicoline or homotaurine may contribute to a reduced cell viability in retinal cells. As shown in Figure 1, retinal cells were well preserved in citicoline- or homotaurine-treated cultures, with no evidence of toxicity or significant loss of viability after treatments. Moreover, it has been previously shown that $100 \mu \mathrm{M}$ of citicoline is not harmful to retinal neuroglial cells in vitro and $100 \mu \mathrm{M}$ of homotaurine is an effective concentration to enhance neuroprotection in a model of experimental glaucoma $[27,28]$. Therefore, this concentration of citicoline and homotaurine was used for all subsequent experiments.

\subsection{Cotreatment of Citicoline and Homotaurine Exerts} Synergistic Effects against Excitotoxic Cell Damage. To evaluate whether cotreatment with citicoline and homotaurine was able to induce a synergistic neuroprotective effect against glutamate excitotoxicity, retinal cell cultures were exposed to citicoline $100 \mu \mathrm{M}$, homotaurine $100 \mu \mathrm{M}$, and citicoline + homotaurine $100 \mu \mathrm{M}, 24$ hours before glutamate treatment. In the presence of $100 \mu \mathrm{M}$ citicoline, a significant increase in cell viability was observed (Figure 2). Although less effective than citicoline in terms of increased cell viability, significant neuroprotection was also observed following treatment with $100 \mu \mathrm{M}$ homotaurine (Figure 2). These data are consistent with previous studies, suggesting the neuroprotective activities of these compounds when used alone [28, 29].

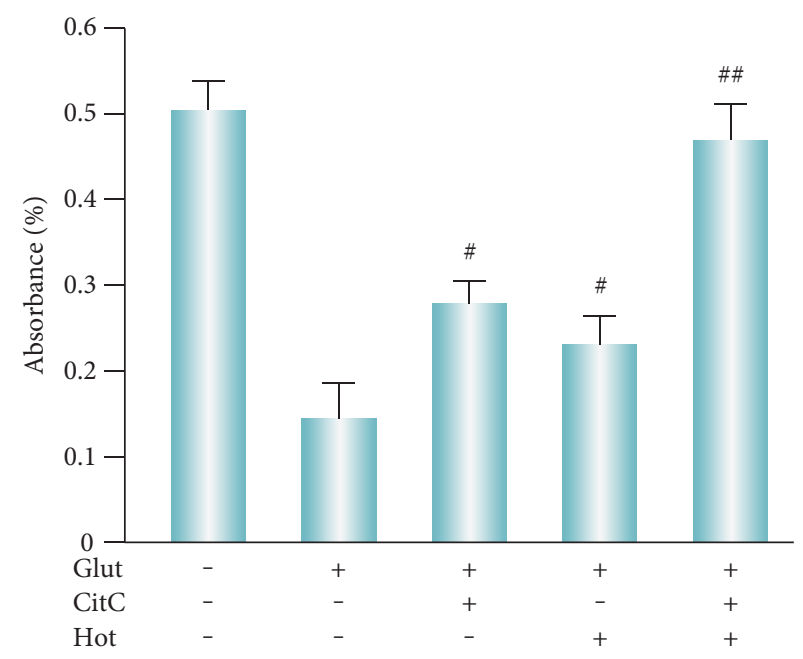

FIgURE 2: Cotreatment of citicoline and homotaurine protects retinal cells against glutamate-induced cytotoxicity. MTT assay was performed to detect cell viability after treatment with CitC and Hot against glutamate-induced cytotoxicity in retinal cells. The combined administration of citcoline and homotaurine demonstrated a significant synergistic cytoprotective effect. The results represent the mean \pm SEM of three independent experiments. ANOVA followed by Tukey's post hoc test was carried out to determine the level of significance. ${ }^{\#} p<0.001$ versus glutamate. ${ }^{\# \#} p<0.001$ versus citicoline and homotaurine alone. CitC: citicoline; Hot: homotaurine; Glut: glutamate.

However, the combination between citicoline and homotaurine significantly increased the viability of retinal cells after glutamate exposure (Figure 2). These results showed that combined administration of citicoline and homotaurine possesses a cytoprotective activity greater than the response achieved by the single compounds.

\subsection{Cotreatment of Citicoline and Homotaurine Reduces} Apoptosis Induced by Administration of Glutamate and HG. Next, we determined whether the synergistic effect of citicoline and homotaurine is associated with cytoprotection against glutamate-induced apoptosis. Apoptosis, measured by the number of oligonucleosomes released, was significantly decreased in cells incubated only with $100 \mu \mathrm{M}$ of citicoline 24 hours before glutamate treatment (Figure 3). Homotaurine $100 \mu \mathrm{M}$ also was able to decrease the neurotoxic effect glutamate in terms of reduction in apoptotic rate (Figure 3). However, as shown in Figure 3, reduction of retinal cell apoptosis induced by these compounds in combination was higher than the groups of either citicoline or homotaurine treated alone. These data suggest that citicoline or homotaurine in combination synergistically reduces apoptosis in glutamate-treated retinal cells. In addition, a neuroprotective effect was also observed against apoptosis induced by HG treatment. Primary retinal cell cultures, exposed to HG treatment, showed an increase in apoptosis, which was reduced in the presence of $100 \mu \mathrm{M}$ citicoline (Figure 4). Significant neuroprotective effects on apoptosis induced by $\mathrm{HG}$ treatment were also reported following treatment with homotaurine $100 \mu \mathrm{M}$ (Figure 4). Again in the 


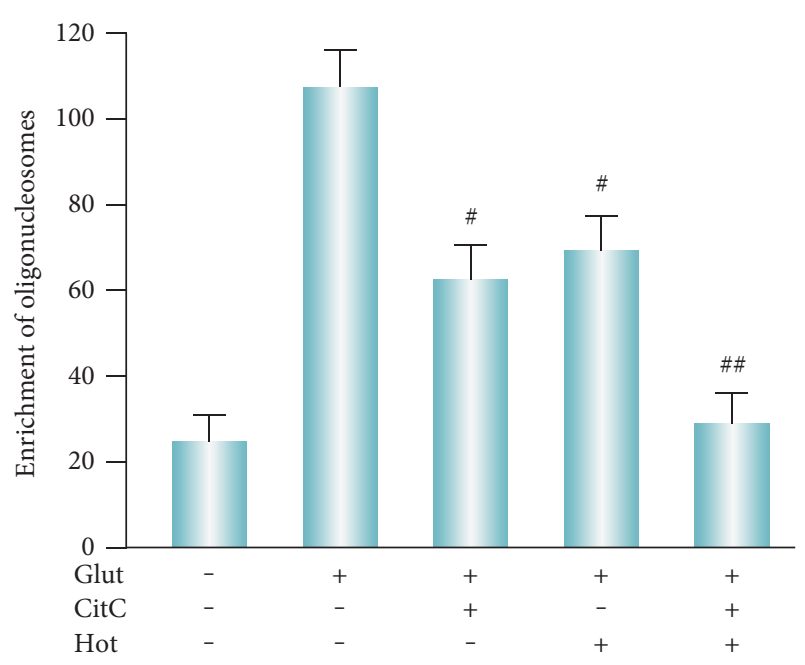

FIGURE 3: Cotreatment of citicoline and homotaurine significantly reduces the apoptotic rate in glutamate-treated cells. A cell death detection ELISA kit was used to determine cytoplasmic DNA oligonucleosome fragments associated with apoptotic cell death. The enrichment of oligonucleosomes released into the cytoplasm was calculated as absorbance of sample cells/absorbance of control cells. The administration of citicoline plus homotaurine demonstrated a synergistic effect in terms of apoptosis reduction. The results represent the mean \pm SEM of three independent experiments. ANOVA followed by Tukey's post hoc test was carried out to determine the level of significance. ${ }^{*} p<0.001$ versus glutamate. ${ }^{\# \#} p<0.001$ versus citicoline and homotaurine alone. CitC: citicoline; Hot: homotaurine; Glut: glutamate.

presence of cotreatment with citicoline and homotaurine, apoptosis was significantly reduced in retinal cell cultures exposed to HG toxicity (Figure 4). Collectively, these results suggested that the enhanced reduction of apoptosis by combination treatment with citicoline and homotaurine may be a useful approach to exert a neuroprotective activity under conditions inducing retinal neurodegeneration.

\section{Discussion}

In this study, we tested synergistic neuroprotective effects of citicoline and homotaurine in combination on primary retinal cells exposed to glutamate toxicity and HG levels. The data demonstrated that cotreatment of citicoline and homotaurine has a direct neuroprotective effect in an experimental model of retinal neurodegeneration. Glutamate-induced excitotoxicity is implicated in the pathophysiology of several degenerative diseases of the retina, including glaucoma. Moreover, HG-induced neurotoxicity is a characteristic of diabetic retinopathy $[30,31]$. Thus, the results of our study provide a rationale for the use of citicoline and homotaurine as potential therapeutic compounds in acute and chronic neurodegenerative diseases of the retina. To our knowledge, this is the first report demonstrating that the neurotoxic effect of glutamate and HG is greatly reduced by simultaneous application of citicoline and homotaurine. Therefore, the neuroprotective activity observed here provides also evidence that combinatorial treatment with

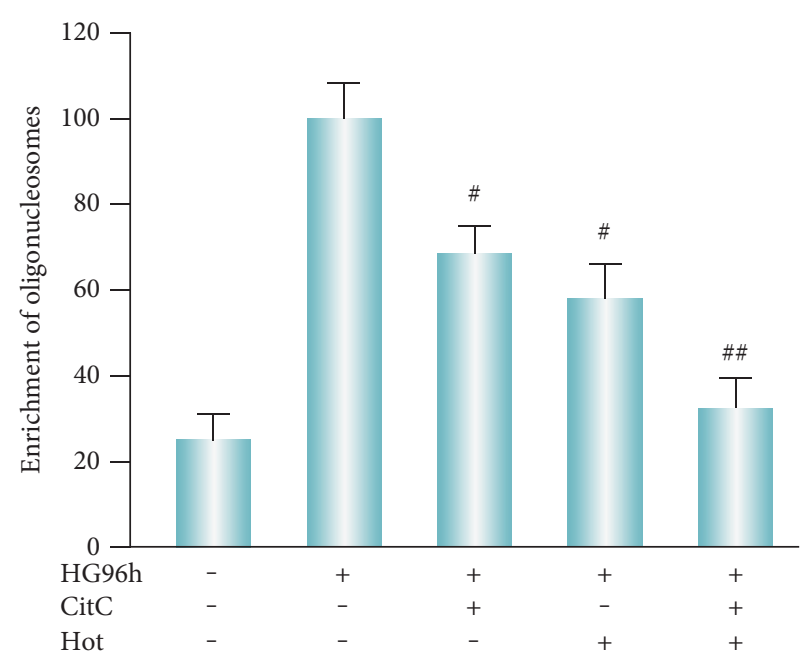

Figure 4: Combined administration of citicoline and homotaurine significantly reduces the apoptotic rate in high glucose-treated cells. Oligonucleosome fragments associated with apoptosis were quantified by cell death detection ELISA. As compared to the untreated cells, the administration of citicoline plus homotaurine demonstrated a statistically significant effect in terms of reduction of oligonucleosome levels. Data of three independent experiments are expressed as mean \pm SEM of the absorbance from treated cells relative to absorbance of untreated cells. Comparison between data sets was performed using ANOVA followed by Tukey's post hoc test. ${ }^{\#} p<0.001$ versus high glucose 96 hours. ${ }^{\# \#} p<0.001$ versus citicoline and homotaurine alone. CitC: citicoline; Hot: homotaurine; HG: high glucose.

these compounds may be a promising strategy to support retinal health. Indeed, an emerging therapeutic approach to counteract neuronal vulnerability associated with aging involves the mixture of distinct compounds, in order to improve the neuroprotective efficacy and pharmacokineticpharmacodynamic properties $[32,33]$. Although the neuroprotective mechanisms of citicoline have been shown in various experimental models of retinal degeneration [34], the presence of homotaurine may increase the neuroprotective effects exerted by this compound. Moreover, it should be highlighted that recent studies have associated the neuroprotective activity of citicoline to its ability in activating sirtuin-1 (SIRT1), a member of the mammalian sirtuins important for neuronal plasticity, cognitive functions, as well as protection against aging-associated neuronal degeneration, and cognitive decline $[17,18]$. The clinical efficacy of homotaurine has been extensively studied in several randomized, double-blind, placebo-controlled phase I, II, and III clinical trials, showing significant positive effects on secondary endpoints in patients with Alzheimer's disease [35, 36]. More interestingly, the association of homotaurine, carnosine, and forskolin (Coleus forskohlii root extract) has shown synergistic neuroprotective effects on RGC both in vitro and in vivo in a mouse model of hypertensive retinal ischemia [23, 37]. Although more than one mechanism might account these synergistic properties, this neuroprotection was associated with reduced calpain activity, upregulation of the phosphoinositide 3-kinase (PI3K)/Akt pathway, and inhibition of 
glycogen synthase kinase- $3 \beta$ (GSK-3 $\beta$ ). Moreover, a recent pilot study demonstrated that oral administration of homotaurine, forskolin, carnosine, and folic acid improves intraocular pressure in patients with primary open-angle glaucoma [38]. Therefore, a multitarget approach by using a combination of molecules may be a more promising strategy to prevent retinal degeneration or slow down glaucomatous progression. In several experimental models of glaucoma, abundant evidence has been provided in which that RGC apoptosis is the earliest form of cell loss of the disease $[39,40]$. Our results show that, following exposure to toxic levels of glutamate and glucose, cotreatment of citicoline and homotaurine reduced apoptosis of primary retinal cells (Figures 3 and 4). Although the neuroprotective mechanism of action of citicoline and homotaurine is not clear at this time, other authors have observed that particularly citicoline may reduce the retinal neuronal apoptosis induced by HG, increasing the expression of endogenous trophic factors such as brainderived neurotrophic factor (BDNF) and ciliary neurotrophic factor (CNTF) that are transiently upregulated as part of the retinal defense responses. In addition, these effects were associated with the reduction of the expression of active forms of caspase-9 and caspase-3 [41, 42]. Alternatively, considering that toxic levels of glutamate and glucose induce an oxidative stress, by increasing reactive oxygen species (ROS), our findings may be also correlated with citicoline and homotaurine antioxidant activities [43,44]. It should be also mentioned that taurine (2-aminoethanesulfonate), homotaurine analogue and one of the most abundant free amino acids in the brain, has been shown to attenuate retinal glial apoptosis in diabetic rats, suggesting an antiapoptotic action. It has also been suggested that taurine prevents glutamate excitotoxicity by increasing glutamate transporter expression, thereby decreasing glutamate levels. In diabetic patients, taurine depletion may be responsible for glaucomatous optic neuropathy, since RGCs are highly dependent on taurine for survival [45-48]. Altogether, the data presented here strongly suggest that citicoline and homotaurine in combination could be a potential new strategy for the prevention and treatment of neurodegenerative diseases, including glaucomatous retinopathy.

\section{Conclusions}

In conclusion, the present study demonstrated that cotreatment of citicoline and homotaurine exhibited synergistic neuroprotective effects on well-known experimental conditions of retinal neurodegeneration. Further studies are needed to clarify the mechanisms responsible for the observed neuroprotective properties, although our data suggest a reduction of apoptosis. Finally, these findings also suggest that cotreatment of citicoline and homotaurine may represent an interesting strategy to achieve neuroprotection in retinal neurodegeneration.

\section{Conflicts of Interest}

The authors have nothing to declare.

\section{Acknowledgments}

The authors would like to express their appreciation to the FB VISION s.r.l. group for excellent technical assistance.

\section{References}

[1] D. Pascolini and S. P. Mariotti, Global Data on Visual Impairments 2010, World Health Organization, Switzerland, Geneva, 2012, http://www.who.int/blindness/GLOBAL DATAFINALforweb.pdf.

[2] R. R. Bourne, J. B. Jonas, S. R. Flaxman et al., "Prevalence and causes of vision loss in high-income countries and in Eastern and Central Europe: 1990-2010," British Journal of Ophthalmology, vol. 98, no. 5, pp. 629-638, 2014.

[3] H. Levkovitch-Verbin, "Retinal ganglion cell apoptotic pathway in glaucoma: initiating and downstream mechanisms," Progress in Brain Research, vol. 220, pp. 37-57, 2015.

[4] T. S. Kern and A. J. Barber, "Retinal ganglion cells in diabetes," Journal of Physiology, vol. 586, no. 18, pp. 4401-4408, 2008.

[5] M. Ankarcrona, J. M. Dypbukt, E. Bonfoco et al., "Glutamateinduced neuronal death: a succession of necrosis or apoptosis depending on mitochondrial function," Neuron, vol. 15, no. 4, pp. 961-973, 1995.

[6] X. X. Dong, Y. Wang, and Z. H. Qin, "Molecular mechanisms of excitotoxicity and their relevance to pathogenesis of neurodegenerative diseases," Acta Pharmacologica Sinica, vol. 30, no. 4, pp. 379-387, 2009.

[7] R. Simó, C. Hernández, and European Consortium for the Early Treatment of Diabetic Retinopathy (EUROCONDOR), "Neurodegeneration in the diabetic eye: new insights and therapeutic perspectives," Trends in Endocrinology and Metabolism, vol. 25, no. 1, pp. 23-33, 2014.

[8] D. R. Tomlinson and N. J. Gardiner, "Glucose neurotoxicity," Nature Reviews Neuroscience, vol. 9, no. 1, pp. 36-45, 2008.

[9] N. G. Hattangady and M. S. Rajadhyaksha, "A brief review of in vitro models of diabetic neuropathy," International Journal of Diabetes in Developing Countries, vol. 29, no. 4, pp. 143-149, 2009.

[10] N. N. Osborne, "Recent clinical findings with memantine should not mean that the idea of neuroprotection in glaucoma is abandoned," Acta Ophthalmologica, vol. 87, no. 4, pp. 450-4, 2009.

[11] S. Davinelli, N. Sapere, M. Visentin, D. Zella, and G. Scapagnini, "Enhancement of mitochondrial biogenesis with polyphenols: combined effects of resveratrol and equol in human endothelial cells," Immunity \& Ageing, vol. 10, no. 1, p. 28, 2012.

[12] S. Davinelli, V. Calabrese, D. Zella, and G. Scapagnini, "Epigenetic nutraceutical diets in Alzheimer's disease," Journal of Nutrition, Health \& Aging, vol. 18, no. 9, pp. 800-805, 2014.

[13] S. Davinelli, M. Maes, G. Corbi, A. Zarrelli, D. C. Willcox, and G. Scapagnini, "Dietary phytochemicals and neuro-inflammaging: from mechanistic insights to translational challenges," Immunity \& Ageing, vol. 13, no. 1, p. 16, 2016.

[14] S. Davinelli, G. Scapagnini, F. Marzatico, V. Nobile, N. Ferrara, and G. Corbi, "Influence of equol and resveratrol supplementation on health-related quality of life in menopausal women: a randomized, placebo-controlled study," Maturitas, vol. 96, pp. 77-83, 2017. 
[15] N. Pescosolido and A. Librando, "Oral administration of an association of forskolin, rutin and vitamins $\mathrm{B} 1$ and $\mathrm{B} 2$ potentiates the hypotonising effects of pharmacological treatments in POAG patients," Clinica Terapeutica, vol. 161, no. 3, pp. e81-e85, 2010.

[16] K. Diederich, K. Frauenknecht, J. Minnerup et al., "Citicoline enhances neuroregenerative processes after experimental stroke in rats," Stroke, vol. 43, no. 7, pp. 1931-1940, 2012.

[17] P. Grieb, "Neuroprotective properties of citicoline: facts, doubts and unresolved issues," CNS Drugs, vol. 28, no. 3, pp. 185-193, 2014.

[18] P. Gareri, A. Castagna, A. M. Cotroneo, S. Putignano, G. De Sarro, and A. C. Bruni, "The role of citicoline in cognitive impairment: pharmacological characteristics, possible advantages, and doubts for an old drug with new perspectives," Clinical Interventions in Aging, vol. 10, pp. 1421-1429, 2015.

[19] T. M. Wright, “Tramiprosate," Drugs of Today, vol. 42, no. 5, pp. 291-298, 2006.

[20] P. Kocis, M. Tolar, J. Yu et al., "Elucidating the A $\beta 42$ anti-aggregation mechanism of action of tramiprosate in Alzheimer's disease: integrating molecular analytical methods, pharmacokinetic and clinical data," CNS Drugs, vol. 31, no. 6, pp. 495-509, 2017.

[21] R. Ward, T. Cirkovic-Vellichovia, F. Ledeque et al., "Neuroprotection by taurine and taurine analogues," Advances in Experimental Medicine and Biology, vol. 583, pp. 299-306, 2006.

[22] H. Yasuyoshi, S. Kashii, S. Zhang et al., "Protective effect of bradykinin against glutamate neurotoxicity in cultured rat retinal neurons," Investigative Ophthalmology \& Visual Science, vol. 41, no. 8, pp. 2273-8, 2000.

[23] S. Kashii, M. Mandai, M. Kikuchi et al., "Dual actions of nitric oxide in N-methyl-D-aspartate receptor-mediated neurotoxicity in cultured retinal neurons," Brain Research, vol. 711, no. 1-2, pp. 93-101, 1996.

[24] S. Kashii, M. Takahashi, M. Mandai et al., "Protective action of dopamine against glutamate neurotoxicity in the retina," Investigative Ophthalmology \& Visual Science, vol. 35, no. 2, pp. 685-695, 1994.

[25] M. Kikuchi, S. Kashii, Y. Honda et al., "Protective action of zinc against glutamate neurotoxicity in cultured retinal neurons," Investigative Ophthalmology \& Visual Science, vol. 36, no. 10, pp. 2048-2053, 1995.

[26] T. Mosmann, "Rapid colorimetric assay for cellular growth and survival: application to proliferation and cytotoxicity assays," Journal of Immunological Methods, vol. 65, no. 1-2, pp. 55-63, 1993.

[27] A. Matteucci, M. Varano, L. Gaddini et al., "Neuroprotective effects of citicoline in in vitro models of retinal neurodegeneration," International Journal of Molecular Sciences, vol. 15, no. 4, pp. 6286-6297, 2014.

[28] R. Russo, A. Adornetto, F. Cavaliere et al., "Intravitreal injection of forskolin, homotaurine, and L-carnosine affords neuroprotection to retinal ganglion cells following retinal ischemic injury," Molecular Vision, vol. 21, pp. 718-729, 2015.

[29] O. Hurtado, I. Lizasoain, and M. Á. Moro, "Neuroprotection and recovery: recent data at the bench on citicoline," Stroke, vol. 42, no. 1, Supplement 1, pp. S33-S35, 2011.

[30] A. J. Barber, "A new view of diabetic retinopathy: a neurodegenerative disease of the eye," Progress in Neuro-
Psychopharmacology and Biological Psychiatry, vol. 27, no. 2, pp. 283-290, 2003.

[31] C. Nucci, R. Russo, A. Martucci et al., "New strategies for neuroprotection in glaucoma, a disease that affects the central nervous system," European Journal of Pharmacology, vol. 787, pp. 119-126, 2016.

[32] S. Davinelli, R. Di Marco, R. Bracale, A. Quattrone, D. Zella, and G. Scapagnini, "Synergistic effect of L-carnosine and EGCG in the prevention of physiological brain aging," Current Pharmaceutical Design, vol. 19, no. 15, pp. 2722-2727, 2013.

[33] R. J. Williams, K. P. Mohanakumar, and P. M. Beart, "Neuronutraceuticals: the path to brain health via nourishment is not so distant," Neurochemistry International, vol. 89, pp. 1-6, 2015.

[34] P. Grieb, A. Jünemann, M. Rekas, and R. Rejdak, "Citicoline: a food beneficial for patients suffering from or threated with glaucoma," Frontiers in Aging Neuroscience, vol. 8, p. 73, 2016.

[35] C. Caltagirone, L. Ferrannini, N. Marchionni, G. Nappi, G. Scapagnini, and M. Trabucchi, "The potential protective effect of tramiprosate (homotaurine) against Alzheimer's disease: a review," Aging Clinical and Experimental Research, vol. 24, no. 6, pp. 580-587, 2012.

[36] P. S. Aisen, S. Gauthier, S. H. Ferris et al., "Tramiprosate in mild-to-moderate Alzheimer's disease - a randomized, double-blind, placebo-controlled, multi-centre study (the Alphase study)," Archives of Medical Science, vol. 7, no. 1, pp. 102-111, 2011.

[37] J. I. Dan and N. N. Osborne, “Attivita neuroprotettiva di una associazione di forskolin, omotaurina e carnosina su cellule ganglionari retiniche in vitro sottoposte a stress ossidativo," Ottica Fisiopatologia, vol. 17, pp. 173-182, 2012.

[38] M. G. Mutolo, G. Albanese, D. Rusciano, and N. Pescosolido, "Oral administration of forskolin, homotaurine, carnosine, and folic acid in patients with primary open angle glaucoma: changes in intraocular pressure, pattern electroretinogram amplitude, and foveal sensitivity," Journal of Ocular Pharmacology and Therapeutics, vol. 32, no. 3, pp. 178-183, 2016.

[39] J. Qu, D. Wang, and C. L. Grosskreutz, "Mechanisms of retinal ganglion cell injury and defense in glaucoma," Experimental Eye Research, vol. 91, no. 1, pp. 48-53, 2010.

[40] F. Schuettauf, R. Rejdak, M. Walski et al., "Retinal neurodegeneration in the DBA/2J mouse-a model for ocular hypertension," Acta Neuropathologica, vol. 107, no. 4, pp. 352-358, 2004.

[41] T. Oshitari, N. Yoshida-Hata, and S. Yamamoto, "Effect of neurotrophic factors on neuronal apoptosis and neurite regeneration in cultured rat retinas exposed to high glucose," Brain Research, vol. 1346, pp. 43-51, 2010.

[42] M. Fiedorowicz, D. Makarewicz, K. I. Stańczak-Mrozek, and P. Grieb, "CDP-choline (citicoline) attenuates brain damage in a rat model of birth asphyxia," Acta Neurobiologiae Experimentalis, vol. 68, no. 3, pp. 389-397, 2008.

[43] R. M. Adibhatla, J. F. Hatcher, and R. J. Dempsey, "Citicoline: neuroprotective mechanisms in cerebral ischemia," Journal of Neurochemistry, vol. 80, no. 1, pp. 12-23, 2002.

[44] S. A. Messina and R. Dawson Jr., "Attenuation of oxidative damage to DNA by taurine and taurine analogs," Advances in Experimental Medicine and Biology, vol. 483, pp. 355-367, 2000.

[45] K. Zeng, H. Xu, M. Mi et al., "Effects of taurine on glial cells apoptosis and taurine transporter expression in retina under 
diabetic conditions," Neurochemical Research, vol. 35, no. 10, pp. 1566-1574, 2010.

[46] X. Yu, Z. Xu, M. Mi et al., "Dietary taurine supplementation ameliorates diabetic retinopathy via anti-excitotoxicity of glutamate in streptozotocin-induced Sprague-Dawley rats," Neurochemical Research, vol. 33, no. 3, pp. 500-507, 2008.

[47] F. Franconi, F. Bennardini, A. Mattana et al., "Plasma and platelet taurine are reduced in subjects with insulin-dependent diabetes mellitus: effects of taurine supplementation," American Journal of Clinical Nutrition, vol. 61, no. 5, pp. 1115-1119, 1995.

[48] M. Merheb, R. T. Daher, M. Nasrallah, R. Sabra, F. N. Ziyadeh, and K. Barada, "Taurine intestinal absorption and renal excretion test in diabetic patients: a pilot study," Diabetes Care, vol. 30 , no. 10, pp. 2652-2654, 2007. 


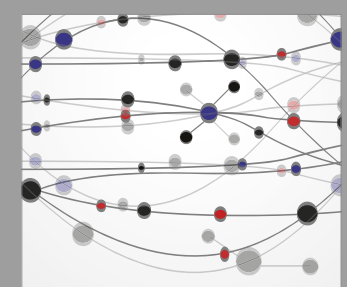

The Scientific World Journal
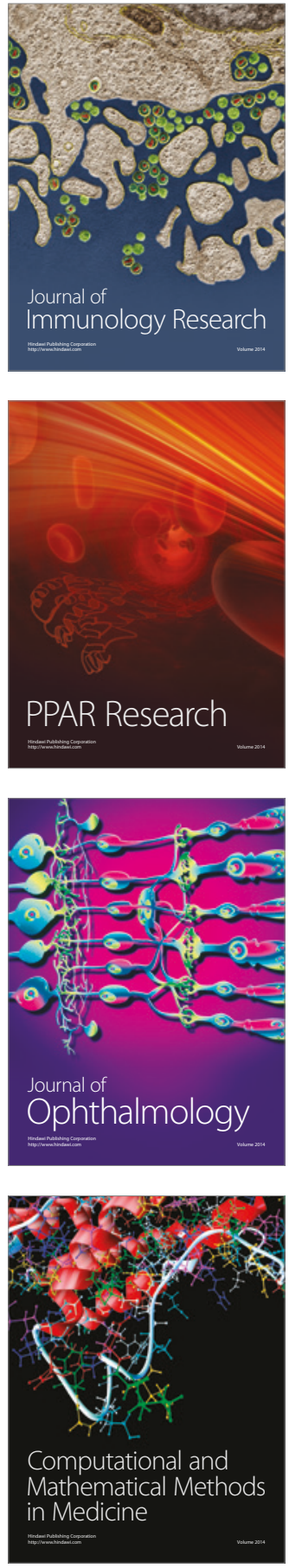

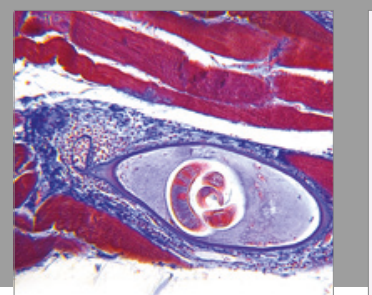

Gastroenterology Research and Practice
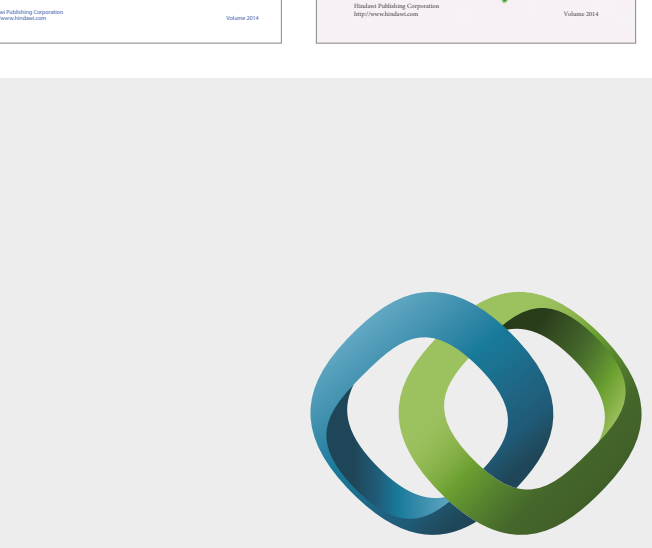

\section{Hindawi}

Submit your manuscripts at

https://www.hindawi.com
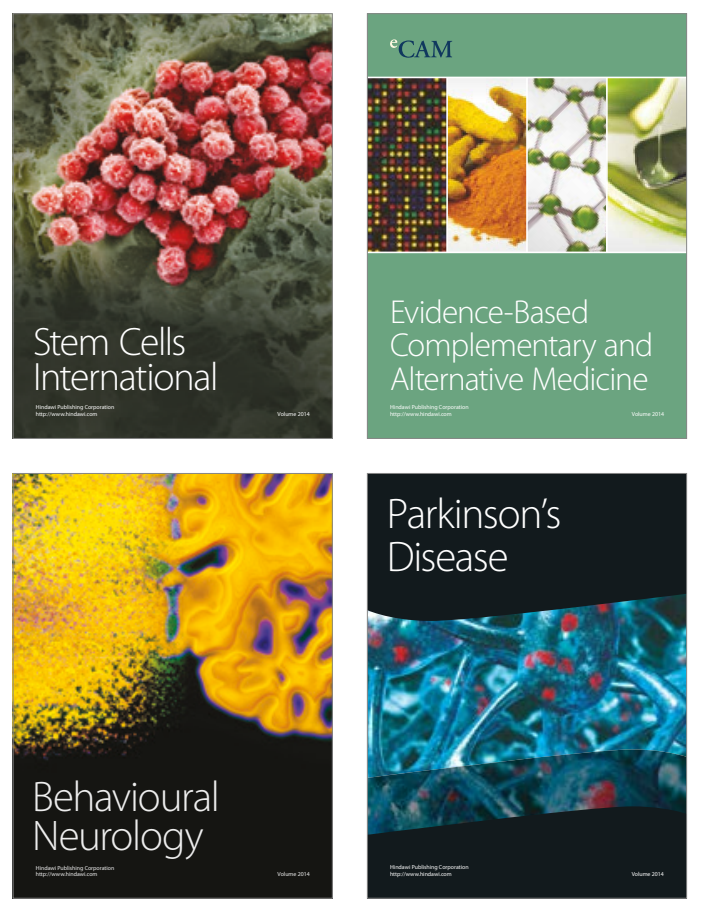
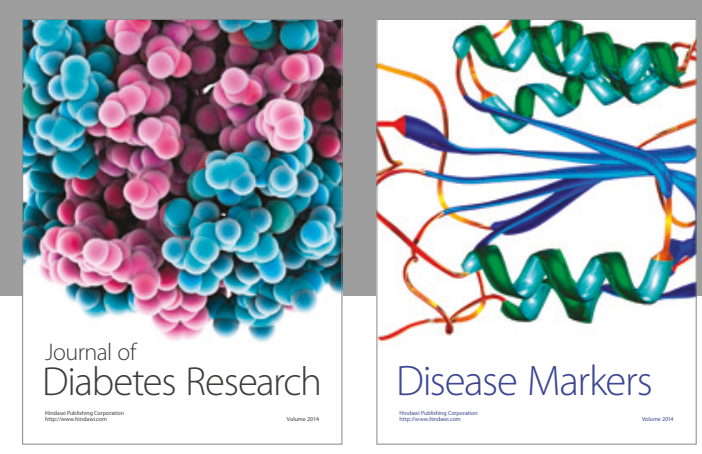

Disease Markers
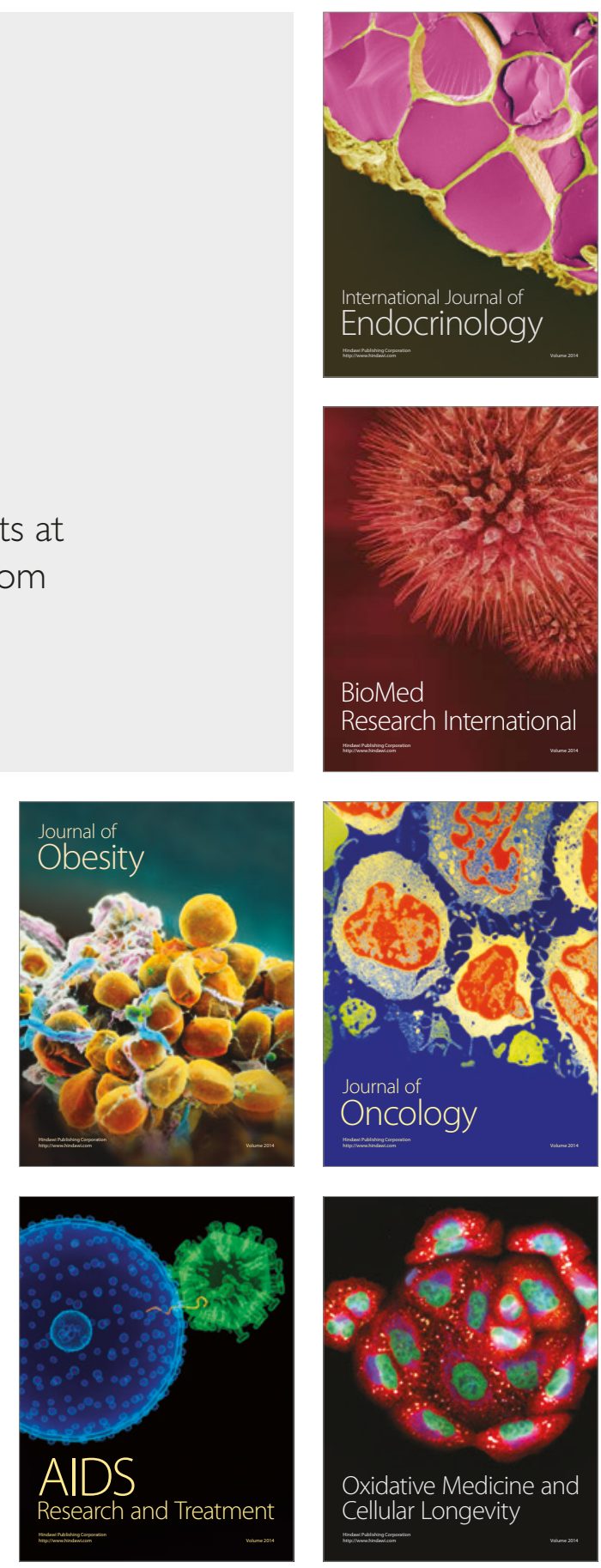